\title{
Optical properties of Ce-doped silica fiber
}

\section{Amit Yadav', Evgeny Zherebtsov's, Nikolay B. Chichkov', Regina Gumenyuk, , Mikhail A. Melkumov', Mikhail V. Yashkov' ${ }^{3}$ Evgeny M. Dianov', and Edik U. Rafailov ${ }^{+}$}

\author{
1. Aston Institute of Photonic Technologies, Aston University, B4 7ET, Birmingham, UK \\ 2. Optoelectronics and measurement techniques unit, University of Oulu, Oulu, 90014, Finland \\ 3. Laboratory of Photonics, Tampere University of Technology, Tampere, Finland \\ 4. Fiber Optics Research Center of the Russian Academy of Sciences, Moscow 119333, Russia \\ 5. Institute of Chemistry of High-Purity Substances of the Russian Academy of Sciences, Nizhny Novgorod 603600, Russia
}

Silica-based luminescent materials have gained significant attention over the last several years primarily to be used under integrated photonics. In this context Ce-doped silica are particularly interesting due to their potential to be used as Si-based light emitting sources [1]. In this work we have prepared a Ce-doped silica fiber that is drawn from a preform which is prepared using standard MCVD technology. In this work we study the spontaneous lifetime and fluorescence parameters of a Ce-doped silica fiber when pumped with $405 \mathrm{~nm}$ continuous wave laser.

The measurements of the spontaneous emission lifetime were implemented in frequency domain by use of the HF2LI $50 \mathrm{MHz}$ lock-in amplifier (Zurich Instruments AG, Switzerland). The fluorescence emission has been excited by $405 \mathrm{~nm}$ laser diode and registered in spectral range of $540 \mathrm{~nm}-700 \mathrm{~nm}$ by use of the scanning monochromator and PMTSS photomultiplier. For that purpose the intensity of excitation radiation was sinusoidally modulated with constant pump modulation factor. This approach allowed us to analyse the decay curves of synchronously detected intensity of the fluorescence intensity and plot its dependence on modulation frequency and the wavelength emission (fig. 1a).

In previous studies it was reported that lifetime $\tau$ for Ce-doped silica can be assessed between 30 and $80 \mathrm{~ns}$ $[2,3]$. Our analysis of the modulation depth of the emission radiation has revealed two components of spontaneous emission with lifetimes $\tau_{1}$ and $\tau_{2}$ respectively. The assessed dependence of the two parameters on emission wavelength is presented in figure $1 \mathrm{~b}$. It should be noted that the $\tau_{2}$ can be explained by the energy level diagrams of Ce-3MR local structure model [3].
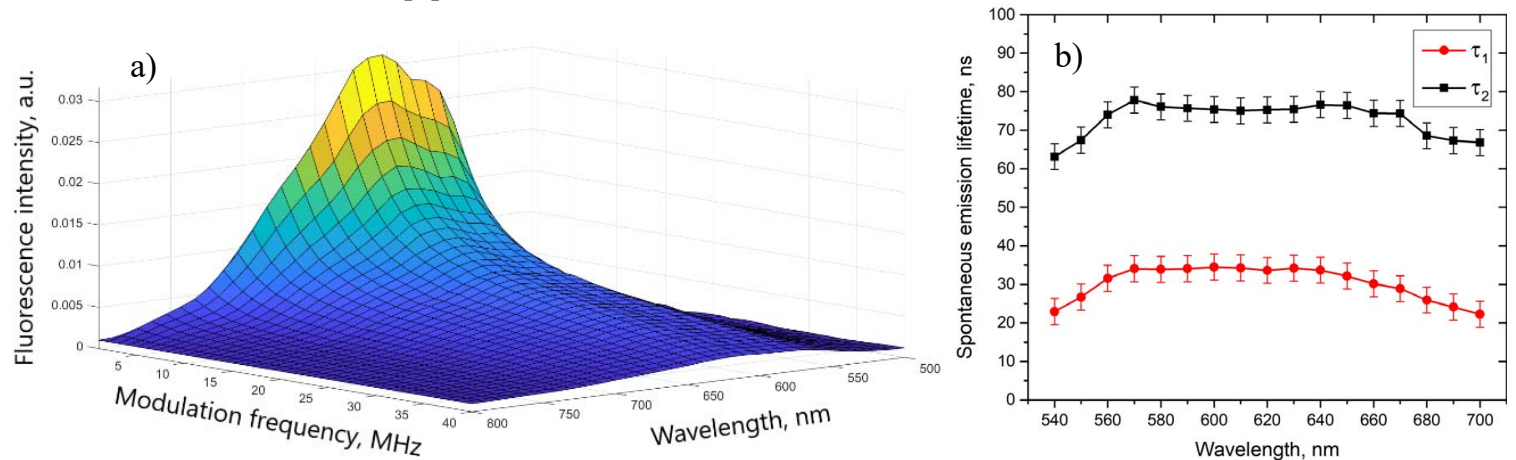

Fig. 1 An example of the dependence of the synchronously detected intensity of the fluorescence on modulation frequency and the wavelength emission (a); dependence of the lifetimes $\tau 1$ and $\tau 2$ of spontaneous emission on emission wavelength

\section{References}

[1] Jing Li, OthmanZalloum, Tyler Roschuk,Chenglin Heng, Jacek Wojcik, Peter Mascher, "The formation of light emitting cerium silicates in cerium-doped silicon oxides," Appl.Phys.Lett., vol. 94, no. 1, pp. 011112, 2009.

[2] X. Sun, J. Wen, Q. Guo, F. Pang, Z. Chen, Y. Luo, G. Peng, and T. Wang, "Fluorescence properties and energy level structure of Cedoped silica fiber materials," Opt. Mater. Express, vol. 7, no. 3, pp. 751-759, 2017.

[3] X. Xu, K. Lebbou, F. Moretti, K. Pauwels, P. Lecoq, E. Auffray, and C. Dujardin, "Ce-doped LuAG single-crystal fibers grown from the melt for high-energy physics," Acta Mater., vol. 67, pp. 232-238, 2014. 